\title{
Oral voriconazole for invasive fungal skull base infection
}

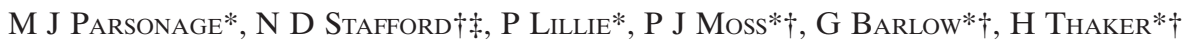

\begin{abstract}
Background: Intravenous amphotericin or intravenous voriconazole, both followed by oral voriconazole, have previously been given to treat invasive aspergillosis of the skull base.

Case report: Exclusively oral voriconazole was used in an immunocompetent patient with biopsy-proven, invasive aspergillosis. She had a large, erosive lesion extending from the central skull base to the right orbit and ethmoid sinus, and displacing the right internal carotid artery. After four months of oral treatment as an out-patient, a repeated computed tomography scan showed a fully treated infection with post-infectious changes only, and treatment was terminated. Two years later, there had been no recurrence.

Conclusion: Substantial cost savings were made by using exclusively oral treatment, compared with the use of intravenous voriconazole or amphotericin, or a switch strategy.
\end{abstract}

Key words: Aspergillosis; Voriconazole

\section{Introduction}

Aspergillus species are a rare cause of skull base masses. Most aspergillus skull base infections occur in immunocompromised patients. Voriconazole is a second generation triazole antifungal agent which is known to penetrate bone, and which has been used successfully in invasive aspergillosis. ${ }^{1,2}$

Here, we present the case of a patient with biopsyproven, invasive aspergillosis involving the skull base and orbit, which responded dramatically to exclusively oral voriconazole therapy.

\section{Case report}

A 31-year-old woman, originally from rural Zimbabwe, was referred to the ear, nose and throat department of her local hospital by a general practitioner and an ophthalmologist. She had previously been well, but complained of a recent three-month history of right-sided proptosis with anosmia and eye discharge. Her vision and cranial nerve functions were intact. Nasal polyps were noted bilaterally.

The patient had an elevated eosinophil count of $0.72 \times$ $10^{9} / 1$ (normal range $0.04-0.4 \times 10^{9} / 1$ ). Her C-reactive protein level was elevated at $26 \mathrm{mg} / \mathrm{l}$ (normal range $0-$ $8 \mathrm{mg} / \mathrm{l}$ ). Serum glucose concentration was normal.

Computed tomography (CT) scanning of the patient's head was performed, which showed a large, erosive lesion extending from the central skull base to the right orbit and ethmoid sinus, and displacing the right internal carotid artery. Foci of calcification were noted in the lesion (Figure 1). Magnetic resonance imaging (MRI) confirmed the findings and suggested a differential diagnosis of chondrosarcoma, fibrous dysplasia or atypical meningioma. A later supplementary report included a fungal lesion as a differential diagnosis due to low signal on MRI, consistent with a high iron content (Figure 2).
Two pernasal biopsies of the right ethmoidal sinus mass were undertaken. Both showed inflammatory tissue rich in eosinophils; one also showed pseudohyphae, which were thought to be due to candida species infection. Fungal serology was performed at this stage for Aspergillus fumigatus subtypes 2109 and 2140, Tinea vulgaris, Microsporum faeni, histoplasma species and cryptococcus species, but was negative. Negative results were also obtained for human immunodeficiency virus antibody and antigen test, syphilis serology, and a $\gamma$-interferon test for Mycobacterium tuberculosis.

A third, open ethmoid biopsy taken via the external route showed fungal hyphae, with the aid of Grocutt's staining. On light microscopy, dichotomous, $45^{\circ}$, branching hyphae, vesicular swellings and a septate appearance were seen, strongly suggestive of aspergillus infection. ${ }^{3}$ Fungal culture was negative; Staphylococcus aureus and klebsiella species were grown but thought to be contaminants.

Following a literature search, oral voriconazole treatment was selected on the grounds of its potential for outpatient treatment, as the patient had a strong preference for this and wished to continue working. Oral voriconazole was given initially at $400 \mathrm{mg}$ twice daily for one day, and then at $200 \mathrm{mg}$ twice daily for four months under close supervision. There were no untoward events. The patient's proptosis resolved almost completely. Her peripheral blood eosinophil count returned to normal, and a second CT scan, performed towards the end of treatment, showed improvement (Figure 3). Subsequent imaging showed that involvement of the right middle cranial fossa had resolved. The floor of the anterior skull base had residual defects but improved ossification, and had the appearance of a fully treated infection.

The patient remained well at the time of writing, with no relapse on 12-month CT (Figure 4). However, she subsequently developed bilateral nasal polyp inflammation.

From the *Departments of Infectious and Tropical Diseases and $\ddagger$ Otolaryngology, Hull and East Yorkshire Hospitals NHS Trust, and $†$ Hull York Medical School, UK.

Accepted for publication: 13 October 2009. First published online 23 December 2009. 


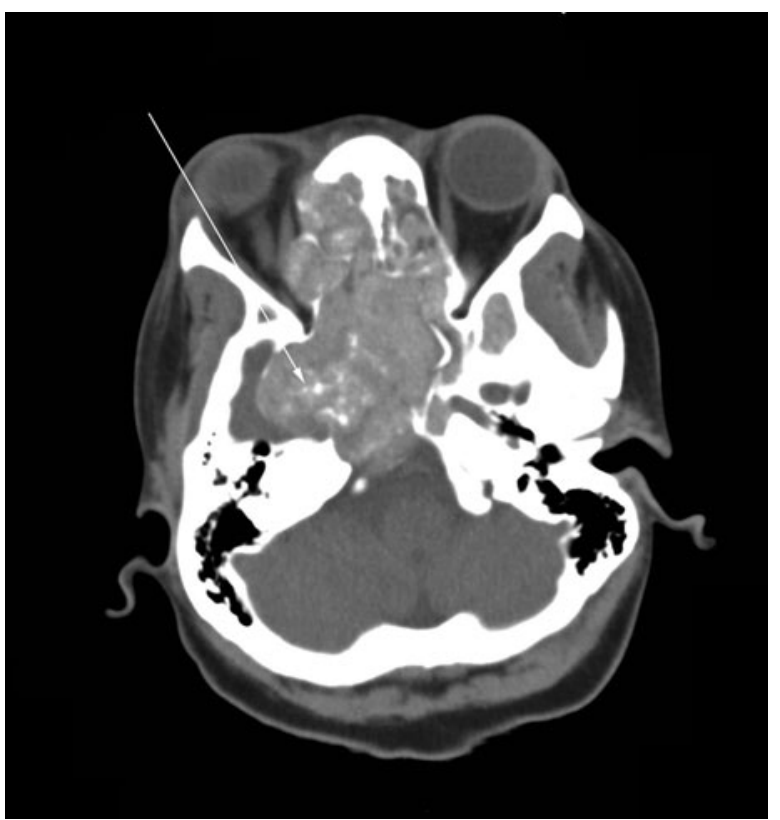

FIG. 1

Axial computed tomography head scan at presentation, showing a mass (arrow) extensively eroding the right-sided and central skull base, including the clivus, right petrous bone and carotid canal, and extending anteriorly to the medial orbital wall.

Biopsy of her left nasal polyp taken eighteen months after completion of voriconazole, showed only inflammatory tissue, and fungal culture was negative.

Due to this patient's impressive result using only oral voriconazole treatment, a further literature search was

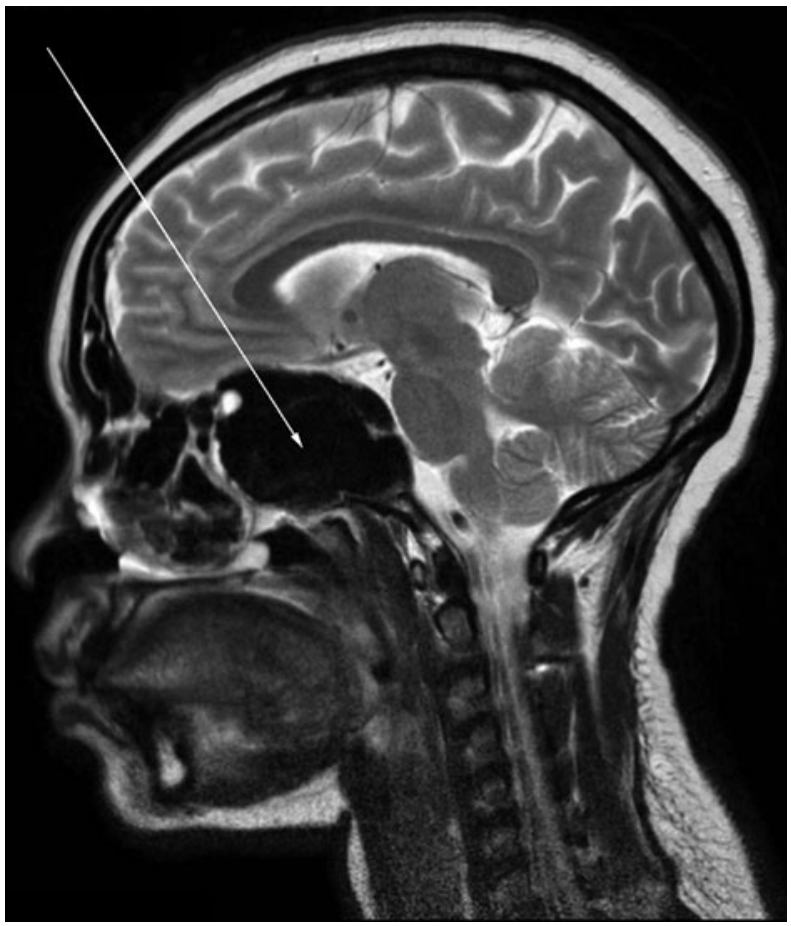

FIG. 2

Sagittal, T2-weighted magnetic resonance imaging brain scan at presentation, showing a low-signal, expansile, sphenoid body mass (arrow).

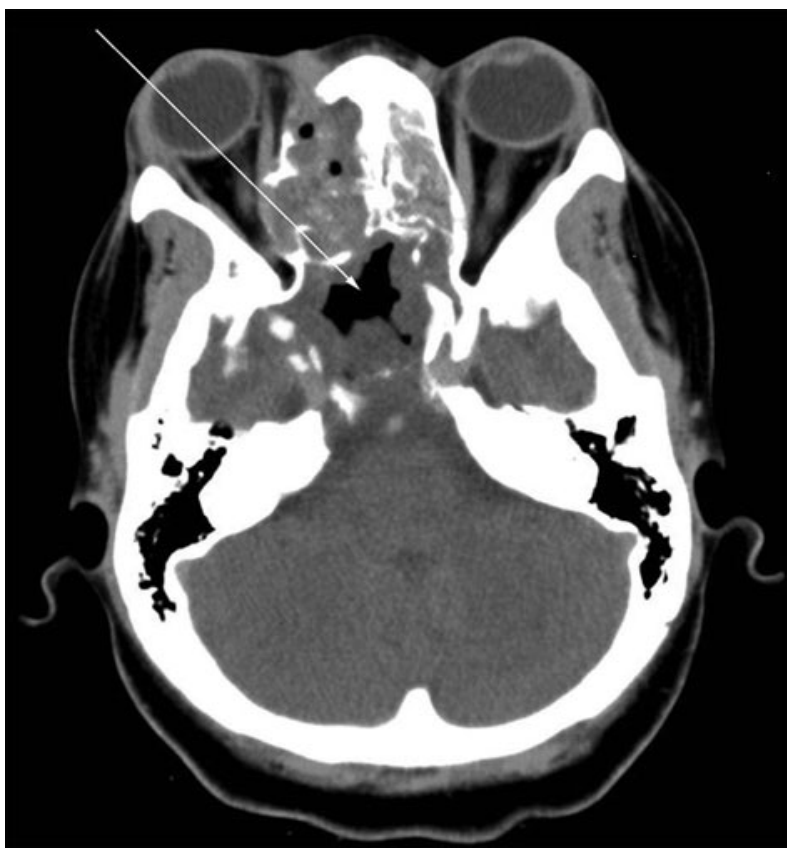

FIG. 3

Axial computed tomography head scan taken after right ethmoid sinus biopsy and near completion of voriconazole treatment, showing the reduced volume of the skull base mass (arrow).

conducted to identify similar cases, using the Medline, Cinahl, Embase and Pubmed databases.

\section{Discussion}

Our immunocompetent patient presented with proptosis, and her CT scan confirmed an erosive sinus lesion. The

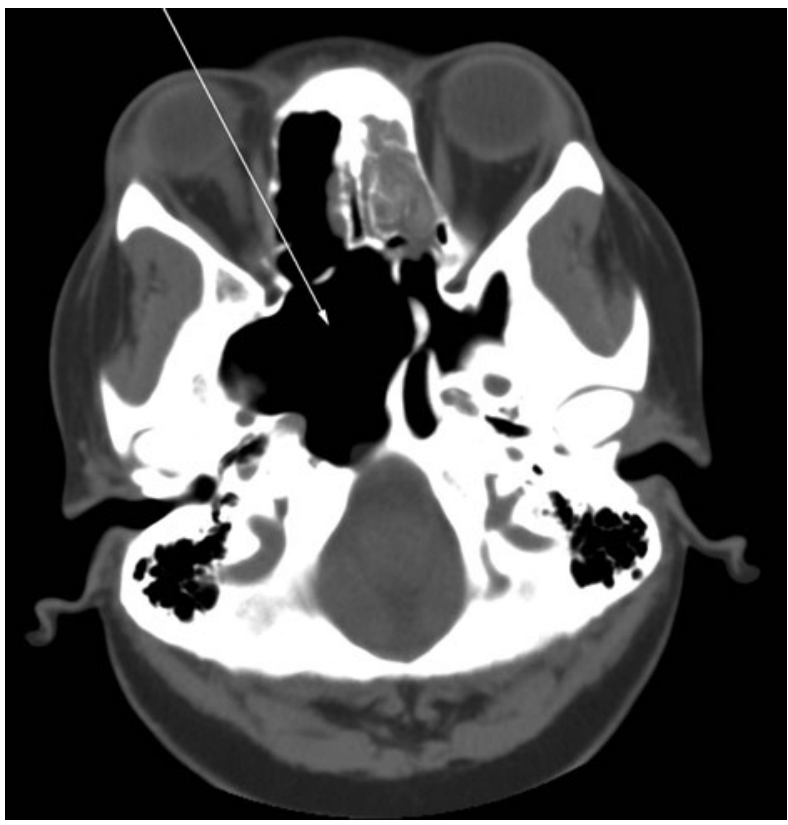

FIG. 4

Axial computed tomography head scan at one year follow up, showing full resolution. Arrow indicates the previous site of the mass. 
final diagnosis by the Criteria of the European Organisation for Research and Treatment of Cancer was histologically proven invasive aspergillus infection with bony invasion and a granulomatous response. ${ }^{4,5}$ She had no history of prior nose or ear disease or dental surgery to indicate a route of entry of the fungus, although nasal polyps were identified (one study found such polyps to be associated with 10 per cent of mycetomas). ${ }^{6}$

Aspergillus is found in soil dust, water and decaying organisms. Inhalation of the spores, usually $A$ fumigatus in Europe, causes fungal sinus infection which may result in local bony invasion. Alternatively, haematogenous spread or entry via the tympanic membrane may occur. ${ }^{7}$

A fungal mass was not suspected radiologically in our patient, and three biopsies were necessary to make the diagnosis. Fungal culture was repeatedly negative; however, other studies have found a low sensitivity of fungal culture in such cases. For example, Klossek et al. found a 30 per cent culture positivity rate and 75 per cent direct fungal smear positivity in a study of 109 mycetomas of the paranasal sinuses. ${ }^{6}$ Other studies have noted a delay in diagnosis and treatment in most cases of invasive aspergillosis of the skull base. ${ }^{8}$ Peripheral blood eosinophilia is well recognised in cases of chronic aspergillus species infection, particularly in the presence of allergy, but also in cases of invasive disease. ${ }^{9}$ In our patient, such eosinophilia may have provided a clue to the correct diagnosis, although there were multiple possible alternative causes in this case.

Previous reports have described itraconazole and amphotericin B as effective treatment for invasive aspergillosis. ${ }^{10}$ Voriconazole was used in our patient because it has increased activity against invasive aspergillosis, compared with fluconazole and amphotericin $\mathrm{B}$, and because the patient expressed a strong desire to be treated as an outpatient. ${ }^{11}$ As a second generation triazole highly active against the enzyme cytochrome P450 14 $\alpha$-demethylase, voriconazole inhibits ergosterol in the cell membrane, disrupting fungal growth. ${ }^{1}$ In a study by Baden et al., predominantly of immunocompromised patients failing or intolerant of standard antifungal therapy, seven of eight patients with central nervous system or sinus disease had a good response to voriconazole. ${ }^{12}$ This is compared to a previously reported, overall treatment response rate of 37 per cent for treatment of aspergillus species infections at various sites. ${ }^{13}$ The known ability of voriconazole to penetrate bone was an additional theoretical advantage; two diabetic but otherwise immunocompetent patients with skull base fungal bone invasion have been reported to have died of neurological sequelae. ${ }^{1,5}$

- Intravenous (IV) amphotericin or voriconazole, with a subsequent IV to oral switch, has been used to treat invasive sinus aspergillosis with extension into the skull base

- This case demonstrates that relatively short courses of oral voriconazole may be effective in selected, carefully monitored cases

- This approach provides considerable cost savings

Previous case reports describing voriconazole treatment of invasive aspergillosis have usually reported initial administration by the intravenous (IV) route, with subsequent switch from IV to oral administration. ${ }^{14}$ However, Swift and Denning have described a case of aspergillus skull base osteitis responding to a 14-month course of oral voriconazole, although this had been preceded by a brief course of itraconazole. ${ }^{15}$ Our patient responded well to an exclusively oral regimen. This is unsurprising, as voriconazole has 96 per cent oral bioavailability. ${ }^{1}$ The use of an oral regimen throughout the treatment course is noteworthy, as oral administration has considerable logistical and financial advantages, is safer, is preferred by patients, and facilitates early discharge from hospital. Since 2006 in the UK, oral voriconazole has cost $£ 79$ per daily dose (the cost is doubled on the first day). Intravenous voriconazole therapy costs approximately double this amount. Lipid formulations of IV amphotericin cost $£ 193 /$ day. Even standard amphotericin costs $£ 12$ per day plus the cost of in-patient care (£252/day in our unit), nursing time, intravenous line placement and daily blood tests. Therefore, our estimated cost savings in this patient were approximately $£ 5100$, based on comparison with IV standard amphotericin for one month as an in-patient with a switch to oral voriconazole thereafter (Hull and East Yorkshire Hospital Trusts finance department, personal communication). ${ }^{16}$ Intravenous amphotericin and voriconazole could have been administered by our outpatient parenteral antibiotic service, but our patient preferred oral therapy, which was also cheaper than this option by $£ 3700$.

\section{Conclusion}

Fungal infections of the skull base occur rarely in immunocompetent patients, but should be included in the differential diagnosis of all skull base mass lesions. The presented case demonstrates that a successful outcome can be achieved with exclusively oral voriconazole therapy in selected, carefully monitored cases of invasive aspergillosis of the skull base in immunocompetent patients.

\section{Acknowledgement}

We thank Dr C Rowland-Hill, Consultant Neuroradiologist at Hull and East Yorkshire NHS Trust, for help with images.

\section{References}

1 Jeu L, Piacenti F, Lyakhovetskiy A, Fung H. Voriconazole. Clin Ther 2003;25:1321-81

2 Ruhnke M. Voriconazole - applications and perspectives. Mycoses 2002;45(Suppl 3):42-7

3 Brandtwein M. Histopathology of sinonasal fungal disease. Otolaryngol Clin North Am 1993;26:949-58

4 De Pauw B, Walsh T, Donnelly P, Stevens D, Edwards J, Calandra $\mathrm{T}$ et al. Revised definitions of invasive fungal disease from the EORTC/MSG Consensus group. Clin Infect Dis 2008;46:1813-21

5 deShazo R, O'Brien M, Chapin K, Soto-Aguilar M, Gardner L, Swain R. A new classification and diagnostic criteria for invasive fungal sinusitis. Arch Otolaryngol Head Neck Surg 1997;123:1181-8

6 Klossek JM, Serrano E, Peloquin L, Percodani J, Fontanel J, Pessey J. Functional endoscopic sinus surgery and 109 mycetomas of paranasal sinuses Laryngoscope 1997:107:112-17

7 Stanley RJ, McCaffrey TV, Weiland LH. Fungal mastoiditis in the immunocompromised host. Arch Otolaryngol Head Neck Surg 1988;114:198-9

8 Hanna E, Hughes G, Eliachar I, Wanamaker J, Tomford W. Fungal osteomyelitis of the temporal bone: a review of reported cases. Ear Nose Throat J 1993;72:532-41

9 Nowicka J, Potoczek S, Jelen M, Chabior M. Aspergillosis of the lymphatic nodes [in Polish]. Pol Tyg Lek 1992;47:812-13

10 Denning DW, Tucker RM, Hanson LH, Stevens DA. Treatment of invasive Aspergillus with itraconazole. Am J Med 1989;86:791-800

11 Patterson TF. Roles of newer azoles in surgical patients. J Chemother 1999;11:504-12

12 Baden LR, Katz JT, Fishman JA, Koziol C, Delvecchio A, Doran $\mathrm{M}$ et al. Salvage therapy with voriconazole for invasive fungal infections in patients failing or intolerant to standard antifungal therapy. Transplantation 2003;76: $1632-7$ 
13 Stevens DA, Kan VL, Judson MA, Morrison VA, Dummer S, Denning DW et al. Practice guidelines for diseases caused by Aspergillus. Clin Infect Dis 2000;30:696-709

14 Amonoo-Kuofi K, Tostevin P, Knight JR. Aspergillus mastoiditis in a patient with SLE: a case report. Skull Base 2005; 15:109-12

15 Swift AC, Denning D. Skull base osteitis following fungal sinusitis. J Laryngol Otol 1998:112:92-7

16 BMJ Group and RPS publishing. British National Formulary. 2006;51

Address for correspondence:

Dr M J Parsonage,
Department of Infectious and Tropical Diseases, Castle Hill Hospital,

Castle Road,

Cottingham,

East Riding HU16 5JG, UK.

Fax: 01482622494

E-mail: mparsonage@btopenworld.com

Dr M Parsonage takes responsibility for the integrity of the content of the paper.

Competing interests: None declared 